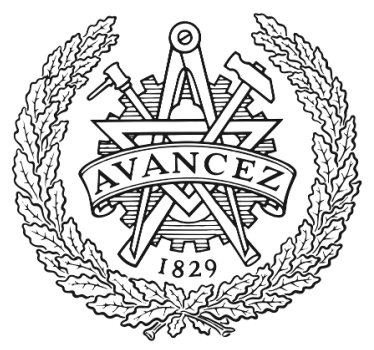

CHALMERS

UNIVERSITY OF TECHNOLOGY

\title{
Mitigation of Ground Clutter in Airborne Bistatic Radar Systems
}

Downloaded from: https://research.chalmers.se, 2023-04-26 12:46 UTC

Citation for the original published paper (version of record):

Klintberg, J., McKelvey, T., Dammert, P. (2020). Mitigation of Ground Clutter in Airborne Bistatic Radar Systems. Proceedings of the IEEE Sensor Array and Multichannel Signal Processing Workshop, 2020-June. http://dx.doi.org/10.1109/SAM48682.2020.9104314

N.B. When citing this work, cite the original published paper. 


\title{
Mitigation of Ground Clutter in Airborne Bistatic Radar Systems
}

\author{
Jacob Klintberg ${ }^{1}$, Tomas McKelvey ${ }^{1}$ and Patrik Dammert ${ }^{1,2}$ \\ ${ }^{1}$ Department of Electrical Engineering, Chalmers University of Technology. Gothenburg, Sweden \\ ${ }^{2}$ Saab BA Surveillance, Saab AB. Gothenburg, Sweden \\ jacobkl@chalmers.se, tomas.mckelvey@chalmers.se, patrik.dammert@ saabgroup.com
}

\begin{abstract}
Space-Time Adaptive Processing is a commonly used technique to mitigate ground clutter reflections from an airborne radar system. It estimates a covariance matrix based on spatial and temporal information, and the estimate is thereafter used to suppress the ground clutter. In a side-looking monostatic radar system, the estimate is rather straight forward based on radar observations. However, in this paper, we consider bistatic systems where the power of adaptivity is limited due to nonstationarity of the ground clutter reflections over the range dimension. To overcome this, scenario dependent transformations are commonly used when forming the sample covariance matrix. In this contribution we instead investigate a detector where the clutter covariance matrix is determined from the geometry of the bistatic scenario. Using a Monte-Carlo simulation, we investigate how sensitive the detector is to errors in the assumed geometry, and compare this with state-of-the-art adaptive methods. The results indicates that a good clutter rejection is obtained for errors of order $10^{3} \mathrm{~m}$ for assumed transmitter position and $10^{\circ}$ $\mathrm{km} / \mathrm{h}$ for assumed transmitter velocity.
\end{abstract}

Index Terms-Space-Time Adaptive Processing, bistatic radar systems, ground clutter mitigation.

\section{INTRODUCTION}

A pulsed Doppler radar is used to detect and track moving objects by measuring objects relative range and velocity compared the radar platform itself. If the radar is equipped with an array antenna, and a train of pulses is transmitted, slow moving targets can be detected from an environment with ground clutter and jammers. If the radar is located on an airborne platform, the ground clutter will have a relative velocity to the radar platform itself. This implies that the ground clutter and possible targets may have a common Doppler frequency. This makes it hard to separate them, and thus the performance of the detector is degraded. To avoid this, the effects of the ground clutter needs to be mitigated. A common technique to suppress the clutter is to use Space-Time Adaptive Processing (STAP).

In a STAP algorithm, spatial and temporal observations are combined to derive data-dependent weights to mitigate the impact of ground clutter and noise. To mitigate the clutter and the noise properly an estimate of their distributions needs to be obtained. The processor characterize the estimate with a covariance matrix, and the estimate is usually based on secondary data and knowledge about antenna array characteristics. However, by using secondary data in the estimate, an assumption is made which presume that the secondary data follows the same distribution as the clutter and the noise in the cell under test. This assumption is not fulfilled for the clutter in several practical applications. A few of these applications are airborne non side-looking antenna arrays, and bistatic radar systems. This paper will further investigate the case of bistatic radar systems.

The secondary data is usually collected from the range dimension. A condition for the STAP algorithm to work properly, is that the distribution of the ground clutter along the range dimension is independent and identically distributed (IID). In such case, the covariance estimate converges to its true value if sufficiently many secondary data is chosen. However, for a bistatic radar scenario, the intensity of the clutter will be range dependent since the geometry between the transmitter, the receiver and the range cell of the secondary data will change depending on the range. This will imply that the secondary data no longer satisfies the assumption of identical distributions over the range dimension. In such scenarios, the secondary data needs to be transformed to satisfy the assumption before an estimate can be obtained.

There exist several algorithms which addresses the problem of range dependent secondary data in an airborne bistatic radar system. Common for most of the algorithms is to analyze, or process, the secondary data in a way such that it appears more identically distributed over range. In a localized processing scheme, secondary data are chosen in a close vicinity to the cell under test to minimize the variations in the secondary data itself [1], [2]. Moreover, in a time-varying weight scheme, the range depending temporal variations are included in the processing. This allows for linear variations of the temporal observations over range [1], [3], [4]. In Doppler warping techniques, the secondary data is homogenized by a transformation which makes the data to appear more similarly distributed over range [5]-[10]. This is done by aligning the angle-Doppler response over several range bins to a common reference point. Several variations of Doppler warping technique exists. For instance, in a registration based method, curve fitting is applied to the direction-Doppler (DD) curve to align different range bins to a common reference DD-curve [9]. While in the Adaptive Angle-Doppler Compensation $\left(\mathrm{A}^{2} \mathrm{DC}\right)$ method, the dominant subspace of the data from different range bins 
are aligned to a common reference subspace [10], [11]. This method aligns the response of the transmitted main lobe, and is completely data dependent. Hence, no knowledge about the velocities and positions of the radar platforms is needed. In [12], a model describing the temporal radar parameters is used to obtain the clutter covariance matrix. This is possible since the clutter distribution is determined by the current radar scenario. Secondary data is then used to fit the model parameters to the temporal observations.

All of the algorithms above obtains an estimate of the ground clutter distribution for the range cell under test based on secondary data from adjacent range bins. To obtain a proper estimate the secondary data is processed to fit the cell under test. However, the ground clutter distribution is deterministically determined by the current radar scenario, which implies that secondary data is not necessary to obtain an estimate of the clutter distribution. Instead, the covariance matrix can be obtained from an assumed radar scenario. The parameters which affects the radar scenario, and hence how the ground clutter will be distributed, are the position and the velocities of the transmitter and receiver platforms, as well as the gain pattern of the transmitter antenna. If all of these parameters are known, the covariance matrix of the ground clutter reflections can be determined. However, in a real application, neither the positions, the velocities nor the gain pattern are exactly known, but will be uncertain to the processor. Particularly quantities connected to the transmitter are uncertain for the receiving platform. This will introduce an error in the assumed radar scenario compared to the true one. Similar ideas are utilized in [12] where secondary data are used to adjust model parameters to a current scenario. However, the usage of several connected models makes the connections between parameter uncertainty and outcome in detector performance hard to interpret. Therefore, in this work, we perform a sensitivity analysis which analyses how a certain level of uncertainty in the assumed radar scenario will effect the performance of the detector.

The paper is organized as follows. In Section II we give a background and introduce signal models for radar signal processing. In Section III we present how the distribution of the ground clutter can be determined from an assumed radar scenario, and in Section IV we evaluate the performance from numerical simulations. Finally, the paper is concluded in Section V.

\section{Signal Model}

Consider an $N$ channel array receiving and processing $M$ pulses over $K$ ranges. The space-time snapshot at range bin $k$ is $\mathbf{x}_{k} \in \mathbb{C}^{M N \times 1}$. We assume that ground clutter $\mathbf{x}_{k, c} \in \mathbb{C}^{M N \times 1}$, receiver noise $\mathbf{x}_{k, n} \in \mathbb{C}^{M N \times 1}$ and possible targets $\mathbf{x}_{k, s} \in$ $\mathbb{C}^{M N \times 1}$ additively comprises the snapshot. To determine if a target is present in range bin $k$, binary hypothesis testing is performed

$$
\begin{aligned}
& H_{0}: \mathbf{x}_{k / H_{0}}=\mathbf{x}_{k, c}+\mathbf{x}_{k, n} \\
& H_{1}: \mathbf{x}_{k / H_{1}}=\mathbf{x}_{k, s}+\mathbf{x}_{k, c}+\mathbf{x}_{k, n},
\end{aligned}
$$

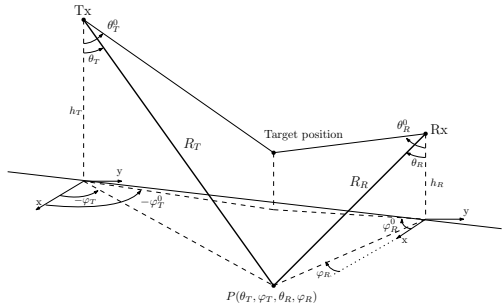

Fig. 1. A general bistatic radar geometry.

where $H_{0}$ is the null hypothesis where no target is present in range bin $k$, and $H_{1}$ is the alternative hypothesis with a target present. To determine between the hypotheses, the elements in $\mathbf{x}_{k}$ are linearly combined to a scalar output $y_{k}=\mathbf{w}_{k}^{H} \mathbf{x}_{k}$. The detector then compares the output with a threshold to determine if a target is present or not.

The purpose of STAP is to suppress clutter, jammers and noise while preserving the desired radar returns. This can be achieved by properly chosing the weights in the scalar output. The optimal weights, from a Signal to Noise Ratio (SNR) perspective, is the vector $\mathbf{w}_{k, \text { opt }}=\mu R_{k}^{-1} \mathbf{s}_{t s}$, where $\mu$ is an arbitrary constant, $\mathbf{s}_{t s}$ is the space-time steering vector and $R_{k}=E\left[\mathbf{x}_{k / H_{0}} \mathbf{x}_{k / H_{0}}^{H}\right] \in \mathbb{C}^{N M \times N M}$ is the nullhypothesis covariance matrix. The superscript ' $H$ ' denotes the Hermitian transpose. Thus, we have that $\mathbf{x}_{k / H_{0}} \sim C N\left(0, R_{k}\right)$. In practice, neither $R_{k}$ nor $\mathbf{s}_{t s}$ is available, and hence needs to be estimated. Substituting $R_{k}$ with its estimate $\hat{R}_{k}$ and $\mathbf{s}_{t s}$ with the hypothesized space-time steering vector $\mathbf{v}_{t s}$, the estimated weight vector becomes $\hat{\mathbf{w}}_{k}=\hat{\mu} \hat{R}_{k}^{-1} \mathbf{v}_{t s}$, where $\hat{\mu}$ is a constant which may depend on the estimated quantities [13].

In Fig 1 a general bistatic geometry is shown. We denote the transmitter as $\mathrm{Tx}$, the receiver as $\mathrm{Rx}$ and the ground clutter patch as $P\left(\theta_{T}, \varphi_{T}, \theta_{R}, \varphi_{R}\right) .\left(\theta_{T}, \varphi_{T}\right)$ and $\left(\theta_{R}, \varphi_{R}\right)$ are elevation and azimuth angles between the transmitter respectively the receiver to the clutter patch. The distance from the transmitter to the clutter patch $P\left(\theta_{T}, \varphi_{T}, \theta_{R}, \varphi_{R}\right)$ is $R_{T}$, and the distance from the receiver to the same clutter patch is $R_{R}$. Hence, the bistatic range from the transmitter and the receiver platforms to a clutter patch is $R=R_{T}+R_{R}$. Clutter patches with the same bistatic range to the transmitter and the receiver platforms will be considered to be in the same range bin. Elevation and azimuth angles, to where the transmitter antenna array is electronically steered, are denoted with $\left(\theta_{T}^{0}, \varphi_{T}^{0}\right)$. The detector in the receiver is searching for possible targets at azimuth and elevation angles $\left(\theta_{R}^{0}, \varphi_{R}^{0}\right)$. It is assumed that the gain pattern for the transmitter is coordinated with the detector in the receiver, hence the transmitted main-lobe and the searched angles intersect with each other. Moreover, both the transmitter and the receiver employ a true north coordinate system, the earth is assumed to be flat, and that the two platforms move at a constant altitude. Also, the scenario is considered to be fixed for all transmitted and received data.

The bistatic Doppler frequency for a clutter patch depends on both the transmitters and the receivers velocities and 
direction of flight, and is given by

$$
\begin{aligned}
f_{d}= & \frac{\left|\mathbf{v}_{T}\right|}{\lambda} \cos \left(\theta_{\mathbf{v}, T}-\theta_{T}\right) \sin \left(\varphi_{\mathbf{v}, T}-\varphi_{T}\right) \\
& +\frac{\left|\mathbf{v}_{R}\right|}{\lambda} \cos \left(\theta_{\mathbf{v}, R}-\theta_{R}\right) \sin \left(\varphi_{\mathbf{v}, R}-\varphi_{R}\right),
\end{aligned}
$$

where $\left|\mathbf{v}_{T}\right|$ and $\left|\mathbf{v}_{R}\right|$ are the transmitter and the receiver speeds, and $\left(\theta_{\mathbf{v}, T}, \theta_{\mathbf{v}, R}\right)$ respectively $\left(\varphi_{\mathbf{v}, T}, \varphi_{\mathbf{v}, R}\right)$ are elevation and azimuth angles for the direction of flight for the transmitter and the receiver. The wavelength of the propagating RF signal is denoted with $\lambda$.

A temporal steering vector characterize the linear phase shifts between the first pulse and the remaining $M-1$ pulses hitting the receiver. The time derivative of this phase function normalized with $1 /(2 \pi)$ gives the Doppler frequency described in (2). The temporal steering vector is

$$
\begin{aligned}
\mathbf{s}_{t}\left(f_{d}\right)= & {\left[\begin{array}{lll}
1 & \exp \left(j 2 \pi f_{d} T\right) & \exp \left(j 2 \pi f_{d} 2 T\right) \\
& \cdots \exp \left(j 2 \pi f_{d}(M-1) T\right)
\end{array}\right]^{T}, }
\end{aligned}
$$

where $T$ is the pulse repetition interval (PRI), and the superscript ' $T$ ' denotes matrix transpose.

In the same way, the varying phase among receiving subarrays for an incoming wave can be described by a steering vector. For a Uniform Linear Array (ULA), the phase variations occurs linear, and the spatial steering vector is

$$
\begin{aligned}
& \mathbf{s}_{s}\left(\theta_{R}, \varphi_{R}\right)=\left[1, \exp \left(j \frac{2 \pi d}{\lambda} \cos \left(\theta_{R}\right) \cos \left(\varphi_{v, R}-\varphi_{R}\right)\right),\right. \\
& \exp \left(j \frac{2 \pi 2 d}{\lambda} \cos \left(\theta_{R}\right) \cos \left(\varphi_{v, R}-\varphi_{R}\right)\right), \\
&\left.\cdots \exp \left(j \frac{2 \pi(N-1) d}{\lambda} \cos \left(\theta_{R}\right) \cos \left(\varphi_{v, R}-\varphi_{R}\right)\right)\right]^{T},
\end{aligned}
$$

where $d$ is the distance between two adjacent subarrays, and the direction of the array is assumed to be parallel with the receiver platform's heading.

The space-time steering vector is given by the Kronecker product between the temporal and the spatial steering vectors. Thus, we have

$$
\mathbf{s}_{t s}\left(\theta_{R}, \varphi_{R}, f_{d}\right)=\mathbf{s}_{t}\left(f_{d}\right) \otimes \mathbf{s}_{s}\left(\theta_{R}, \varphi_{R}\right) .
$$

The bistatic ground clutter response for a range bin is the continuous voltage response from all scatters comprising the range resolution cell. The ground clutter snapshot for range bin $k$ can be modeled by [14]

$$
\begin{aligned}
\mathbf{x}_{k, c}= & \int_{R-\Delta_{R} / 2}^{R+\Delta_{R} / 2} \int_{0}^{2 \pi} \sigma_{c}\left(\theta_{R}, \varphi_{R} \mid \theta_{T}, \varphi_{T}, \theta_{T}^{0}, \varphi_{T}^{0}\right) \\
& \cdot v\left(\theta_{R}, \varphi_{R} \mid \theta_{T}, \varphi_{T}\right) \mathbf{s}_{t s}\left(\theta_{R}, \varphi_{R}, f_{d}\right) d \theta_{R} d r
\end{aligned}
$$

where $\Delta_{R}$ defines the bound between two adjacent range bins, $\sigma_{c}\left(\theta_{R}, \varphi_{R} \mid \theta_{T}, \varphi_{T}, \theta_{T}^{0}, \varphi_{T}^{0}\right)$ is the intensity of the received observations given the transmitted gain pattern and azimuth and elevation angles for the transmitter and the receiver, and $v\left(\theta_{R}, \varphi_{R} \mid \theta_{T}, \varphi_{T}\right) \sim C N(0,1)$. A discrete approximation of (6), suitable for numerical simulations, is given by:
1. Divide the earth's surface into fractions of a radar resolution cell, and identify all fractions corresponding to the same bistatic range.

2. Calculate the fraction snapshot by muliplying the root pixel power by a standard normal random variable and the appropriate space-time steering vector.

3. Sum all snapshots for a common bistatic range.

The accuracy of the approximation of (6) increases with decreased size of each ground clutter fraction.

\section{DERIVATION OF COVARIANCE MATRIX}

To be able to suppress the ground clutter properly, an estimate of the null hypothesis distribution must be obtained. It is assumed that the null hypothesis is Gaussian distributed with zero mean, therefore the corresponding covariance matrix is sufficient to fully describe the distribution.

Given that the ground clutter reflections for range bin $k$ are given by (6), the clairvoyant covariance matrix for range bin $k$ can be obtained from

$$
\begin{aligned}
R_{k, c}= & \int_{R-\Delta_{R} / 2}^{R+\Delta_{R} / 2} \int_{0}^{2 \pi} \sigma_{c}^{2}\left(\theta_{R}, \varphi_{R} \mid \theta_{T}, \varphi_{T}, \theta_{T}^{0}, \varphi_{T}^{0}\right) \\
& \cdot \mathbf{s}_{t s}\left(\theta_{R}, \varphi_{R}, f_{d}\right) \mathbf{s}_{t s}^{H}\left(\theta_{R}, \varphi_{R}, f_{d}\right) d \theta_{R} d r
\end{aligned}
$$

As can be seen in (7), the covariance matrix depends on the true radar scenario, i.e. the position and velocities of the transmitter and the receiver, and the transmitted gain pattern. In (7), the positions and the velocities are implicitly given by $\theta_{T}, \theta_{R}, \varphi_{T}, \varphi_{R}$ and $f_{d}$.

In a real application, the radar scenario is not perfectly known to the processor. That is, the position and the velocities of the transmitter and the receiver, and the transmitted gain pattern are only known to the processor up to some uncertainty. Especially quantities connected to the transmitter are normally more uncertain to the processor, since the processing is usually performed in the receiver. Therefore, by assuming that the processor has exact knowledge of the parameters connected to the receiver, and that parameters connected to the transmitter are uncertain to the processor. A certainty of equivalence estimate of the covariance matrix is

$$
\begin{aligned}
\hat{R}_{k, c}= & \int_{R-\Delta_{R} / 2}^{R+\Delta_{R} / 2} \int_{0}^{2 \pi} \sigma_{c}^{2}\left(\theta_{R}, \varphi_{R} \mid \hat{\theta}_{T}, \hat{\varphi}_{T}, \hat{\theta}_{T}^{0}, \hat{\varphi}_{T}^{0}\right) \\
& \cdot \mathbf{s}_{t s}\left(\theta_{R}, \varphi_{R}, \hat{f}_{d}\right) \mathbf{s}_{t s}^{H}\left(\theta_{R}, \varphi_{R}, \hat{f}_{d}\right) d \theta_{R} d r
\end{aligned}
$$

where $\left(\hat{\theta}_{T}, \hat{\varphi}_{T}, \hat{\theta}_{T}^{0}, \hat{\varphi}_{T}^{0}, \hat{f}_{d}\right)$ are, by the receiver assumed, parameters of $\left(\theta_{T}, \varphi_{T}, \theta_{T}^{0}, \varphi_{T}^{0}, f_{d}\right)$. The accuracy of the assumed parameters will determine the accuracy of the estimate $\hat{R}_{k, c}$.

\section{NUMERICAL SIMULATIONS}

In this section, we consider numerical simulations to evaluate the performance of the estimated covariance matrix from an assumed radar scenario. The method is compared with the $\mathrm{A}^{2} \mathrm{DC}$ method [10], [11].

The radar scenario that is considered in the simulations is presented in Fig 2 and the radar parameters used in the simulation can be find in Table I. The estimate from the $\mathrm{A}^{2} \mathrm{DC}$ 


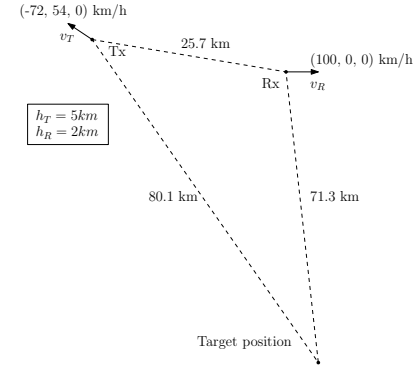

Fig. 2. The bistatic radar scenario considered in the numerical simulations.

TABLE I

RADAR PARAMETERS

\begin{tabular}{|c|c|}
\hline Parameter & Value \\
\hline Number of pulses, $M$ & 16 \\
Number of receive channels, $N$ & 18 \\
Number of transmitting channels & 18 \\
Distance between adjacent antenna arrays & $0.5 \lambda$ \\
Pulse repetition interval & $5 \mathrm{~ms}$ \\
Wavelength of transmitted pulses & $0.05 \mathrm{~m}$ \\
\hline
\end{tabular}

method is based on secondary data, generated according to (6), from adjacent range bins to the cell under test. To follow the RBM rule, $2 N M$ range bins are considered [15]. Moreover, the secondary data is homogenized towards a common reference range bin, which is selected to be the range bin under test. Notice though that the range bin corresponding to the cell under test is not part of the secondary data. The calculation of the covariance matrix from the proposed method is not based upon secondary data, but is calculated to coincide with the range bin corresponding to the cell under test.

The methods are compared by evaluating how well their estimate of the ground clutter covariance matrix performs in a radar detector [16]. A target, with velocity $(45,70,0) \mathrm{km} / \mathrm{h}$, is placed in the cell under test to obtain a suitable measure. This implies that target position corresponds to the analysed cell. From the detector, the performance of the hypothesis testing can be evaluated by studying the Receiver Operating Characteristic (ROC) [17]. Moreover, a valid scalar value of the performance can be obtained from the Area Under the Curve (AUC) to the ROC-curve [17].

To obtain a proper AUC measurement for the proposed method, we consider Monte-Carlo simulations. In each MonteCarlo iteration, a random sample for the assumed transmitter position, velocity and direction of gain pattern is considered. The random samples are drawn from

$$
\begin{aligned}
\hat{\mathbf{Q}}_{T x} & =\mathbf{Q}_{T x}+\varepsilon_{\mathbf{Q}_{T x}} \\
\hat{\mathbf{v}}_{T} & =\mathbf{v}_{T}+\varepsilon_{\mathbf{v}_{T}}
\end{aligned}
$$

where the position of the transmitter is denoted $\mathbf{Q}_{T x}, \varepsilon_{\mathbf{Q}_{T x}} \sim$ $\mathcal{N}\left(0, R_{\mathbf{Q}_{T x}}\right)$ and $\varepsilon_{\mathbf{v}_{T}} \sim \mathcal{N}\left(0, R_{\mathbf{v}_{T}}\right)$. The sensitivity of the estimate (8) is analyzed by varying $R_{\mathbf{Q}_{T x}}$ and $R_{\mathbf{v}_{T}}$.

In Fig 3 the corresponding ROC-curves for the clairvoyant detector, the estimate from the $\mathrm{A}^{2} \mathrm{DC}$ method, and the mean ROC-curve for estimates from (8) where the assumed radar parameters are 100 samples from $\hat{\mathbf{Q}}_{T x} \sim \mathcal{N}\left(\mathbf{Q}_{T x}, 10^{3} \cdot \mathbf{I}_{3}\right)$

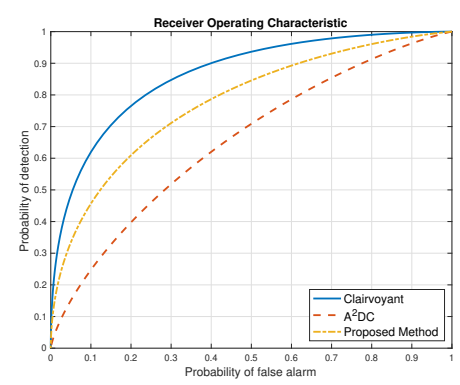

Fig. 3. ROC-curve of detector for the cell under test with clairvoyant covariance matrix, and estimated covariance matrices from the proposed method and $\mathrm{A}^{2} \mathrm{DC}$

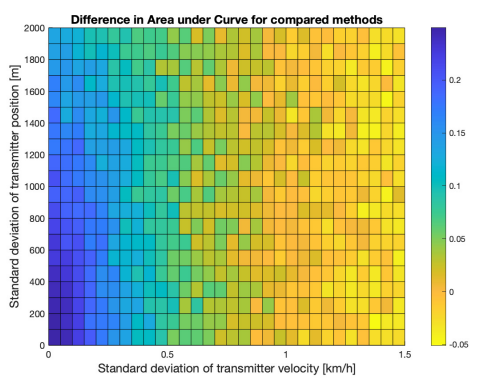

Fig. 4. Difference in AUC of estimates from the proposed method for different assumed radar parameters and the $\mathrm{A}^{2} \mathrm{DC}$ method.

m and $\hat{v}_{T} \sim \mathcal{N}\left(\mathbf{v}_{T}, 0.5 \cdot \mathbf{I}_{3}\right) \mathrm{km} / \mathrm{h}$, is shown. It can be seen that the proposed method gives a better estimate of the clutter covariance matrix than the $\mathrm{A}^{2} \mathrm{DC}$ method.

In Fig 4, the AUC for different assumed transmitter parameters is viewed. The figure shows the difference in AUC between the estimate from (8) and the estimate from the $\mathrm{A}^{2} \mathrm{DC}$ method. Thus, positive values in the figure means that the proposed method results in a better detector, and negative values means that $\mathrm{A}^{2} \mathrm{DC}$ gives a better estimator. Each value for the estimate from (8) is the mean value of the AUC from 100 Monte-Carlo iterations. It should also be noted that the origin in the figure corresponds to the clairvoyant detector. From the results in the example, we note that even for rather large errors in the assumed radar scenario the estimate from (8) still produces a detector which is better than the one from $\mathrm{A}^{2} \mathrm{DC}$.

\section{CONClusion}

This paper consider ground clutter mitigation for airborne bistatic radar systems. The covariance matrix of the ground clutter is determined from the radar geometry, and the transmitted gain pattern. This paper investigate a method which calculates the corresponding covariance matrix from assumed knowledge of the current radar parameters. This implies that no secondary data are necessary in the estimated covariance matrix. The numerical examples indicates an improved clutter rejection compared to other state-of-the-art methods for errors of order $10^{3} \mathrm{~m}$ and $10^{0} \mathrm{~km} / \mathrm{h}$ in the assumed transmitter position and transmitter velocity. 


\section{REFERENCES}

[1] W. L. Melvin, M. J. Callahan, and M. C. Wicks, "Adaptive clutter cancellation in bistatic radar," in Conference Record of the ThirtyFourth Asilomar Conference on Signals, Systems and Computers (Cat. No.00CH37154), vol. 2, Oct 2000, pp. 1125-1130 vol.2.

[2] B. Himed, J. H. Michels, and Yuhong Zhang, "Bistatic stap performance analysis in radar applications," in Proceedings of the 2001 IEEE Radar Conference (Cat. No.01CH37200), May 2001, pp. 198-203.

[3] S. D. Hayward, "Adaptive beamforming for rapidly moving arrays," in Proceedings of International Radar Conference, Oct 1996, pp. 480-483.

[4] S. M. Kogon and M. A. Zatman, "Bistatic stap for airborne radar systems," in Proc. ASAP, 2000, pp. 1-6.

[5] W. L. Melvin, M. J. Callahan, and M. C. Wicks, "Bistatic stap: application to airborne radar," in Proceedings of the 2002 IEEE Radar Conference (IEEE Cat. No.02CH37322), April 2002, pp. 1-7.

[6] G. K. Borsari, "Mitigating effects on stap processing caused by an inclined array," in Proceedings of the 1998 IEEE Radar Conference, RADARCON'98. Challenges in Radar Systems and Solutions (Cat. No.98CH36197), May 1998, pp. 135-140.

[7] F. Pearson and G. Borsari, "Simulation and analysis of adaptive interference suppression for bistatic surveillance radars," MASSACHUSETTS INST OF TECH LEXINGTON LINCOLN LAB, Tech. Rep., 2001.

[8] B. Himed, Y. Zhang, and A. Hajjari, "Stap with angle-doppler compensation for bistatic airborne radars," in Proceedings of the 2002 IEEE Radar Conference (IEEE Cat. No.02CH37322), April 2002, pp. 311317.

[9] F. D. Lapierre, J. G. Verly, and M. Van Droogenbroeck, "New solutions to the problem of range dependence in bistatic stap radars," in Proceed ings of the 2003 IEEE Radar Conference (Cat. No. 03CH37474), May 2003, pp. 452-459.

[10] W. L. Melvin, B. Himed, and M. E. Davis, "Doubly adaptive bistatic clutter filtering," in Proceedings of the 2003 IEEE Radar Conference (Cat. No. 03CH37474), May 2003, pp. 171-178.

[11] W. L. Melvin and M. E. Davis, "Adaptive cancellation method for geometry-induced nonstationary bistatic clutter environments," IEEE Transactions on Aerospace and Electronic Systems, vol. 43, no. 2, pp. 651-672, April 2007.

[12] A. G. Jaffer, B. Himed, and P. T. Ho, "Estimation of range-dependent clutter covariance by configuration system parameter estimation," in IEEE International Radar Conference, 2005., 2005, pp. 596-601.

[13] I. S. Reed, J. D. Mallett, and L. E. Brennan, "Rapid convergence rate in adaptive arrays," IEEE Transactions on Aerospace and Electronic Systems, vol. AES-10, no. 6, pp. 853-863, Nov 1974.

[14] J. Ward, "Space-time adaptive processing for airborne radar," in 1995 International Conference on Acoustics, Speech, and Signal Processing, vol. 5, May 1995, pp. 2809-2812 vol.5.

[15] L. E. Brennan and L. S. Reed, "Theory of adaptive radar," IEEE Transactions on Aerospace and Electronic Systems, vol. AES-9, no. 2, pp. 237-252, March 1973.

[16] A. De Maio and M. Greco, Modern Radar Detection Theory, ser Electromagnetics and Radar. Institution of Engineering and Technology, 2016.

[17] T. Fawcett, "An introduction to roc analysis," Pattern Recognition Letters, vol. 27, no. 8, pp. $861-874$, 2006, rOC Analysis in Pattern Recognition. 